\title{
Energetic electrons in the exterior cusp: identifying the source
}

\author{
B. M. Walsh, T. A. Fritz, M. M. Klida, and J. Chen \\ Center for Space Physics, Boston University, Boston, MA, USA \\ Received: 12 January 2010 - Revised: 8 April 2010 - Accepted: 12 April 2010 - Published: 16 April 2010
}

\begin{abstract}
A case study is presented to test the role several different sources may play in populating the exterior cusp with energetic $(E>40 \mathrm{keV})$ electrons. The properties of the electrons measured in the exterior cusp are compared with the expected signatures of three sources in order to weigh the role each source may contribute in populating this region. The potential sources probed are: (1) solar energetic electrons entering the cusp from the solar wind; (2) an equatorial magnetospheric source with particles drifting to high latitudes on the dayside; and (3) a local source accelerating the electrons in situ. From the observations it is likely that local acceleration is the primary source of the energetic electrons in the exterior cusp during this event.
\end{abstract}

Keywords. Magnetospheric physics (Energetic particles, trapped; Magnetopause, cusp, and boundary layers; Magnetosheath)

\section{Introduction}

The magnetospheric cusps allow for the most direct entry of shocked solar wind plasma into the magnetosphere (Frank, 1971; Heikkila and Winningham, 1971). In order to maintain a pressure balance with adjacent magnetospheric regions, the magnetic pressure in the exterior cusp must decrease to account for the increase in thermal pressure from the newly injected plasma. The result is a diamagnetic cavity in the exterior cusp. In some cases this can bring the magnetic field strength close to $0 \mathrm{nT}$. This magnetic signature has been confirmed through many studies (Paschmann et al., 1976; Fairfield and Ness, 1972; Farrell and Van Allen, 1990). The spatial extent of this diamagnetic region has been measured to be up to $6 R_{\mathrm{E}}$ (Fritz et al., 2003). Hereafter we will refer to the cusp diamagnetic cavity as "CDC."

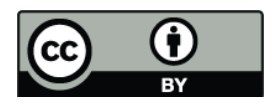

Correspondence to: B. M. Walsh (bwalsh@bu.edu)
The CDC often hosts strong wave activity ranging from ultra low frequencies (Chen and Fritz, 1998; Le et al., 2001) up to the electron cyclotron plasma frequencies (Pickett et al., 2001; Khotyaintsev et al., 2004). The waves have been shown to be both right and left-handed polarized (Nykyri et al., 2004). Large fluxes of energetic ions and electrons, with energies up to $\mathrm{MeV}$ and hundreds of $\mathrm{keV}$ respectively, have also been observed in the exterior cusp. Zhang et al. (2005) has shown through a statistical study with Cluster that these energetic populations are a common feature. Ions with $E>28 \mathrm{keV}$ are present $80 \%$ of the time while electrons with $E>40 \mathrm{keV}$ are present on $22.5 \%$ of exterior cusp crossings (Zhang et al., 2005). Pitch angle distributions of the energetic populations have shown these particles are primarily trapped (Chen et al., 1997; Sheldon et al., 1998), but can also show flows traveling along field lines out of the cusp (Whitaker et al., 2006; Walsh et al., 2007). The origin of the energetic ions observed in the exterior cusp has been a topic of debate in the literature.

Chen and Fritz (1998) associated the presence of energetic ions with ULF wave intensity in the cusp and proposed a resonance source energizing the population within the exterior cusp. Further studies of wave properties in the CDC (Vogiatzis et al., 2008; Chen, 2008) have shown the conditions are often sufficient to energize both ions and electrons up to the observed energies. Additional evidence for local acceleration was provided by Chen and Fritz (2001) and Fritz et al. (2003) who showed the energetic population was composed of both ionospheric $\left(\mathrm{O}^{+}\right)$as well as solar wind $\left(\mathrm{He}^{++}\right.$, $\mathrm{O}^{>+3}$ ) ions. The presence of both species with similar energies and intensity variability with time indicate a common source. Pitch angle distributions of energetic ions trapped and flowing out of the cusp are also indicators of local acceleration being the primary source (Whitaker et al., 2006; Walsh et al., 2007).

In contrast, Chang et al. (1998) proposed ions accelerated at the quasi-parallel bow shock are the dominant source for the energetic ions in the exterior cusp. Magnetic field lines have been traced from the quasi-parallel bow shock to the

Published by Copernicus Publications on behalf of the European Geosciences Union. 


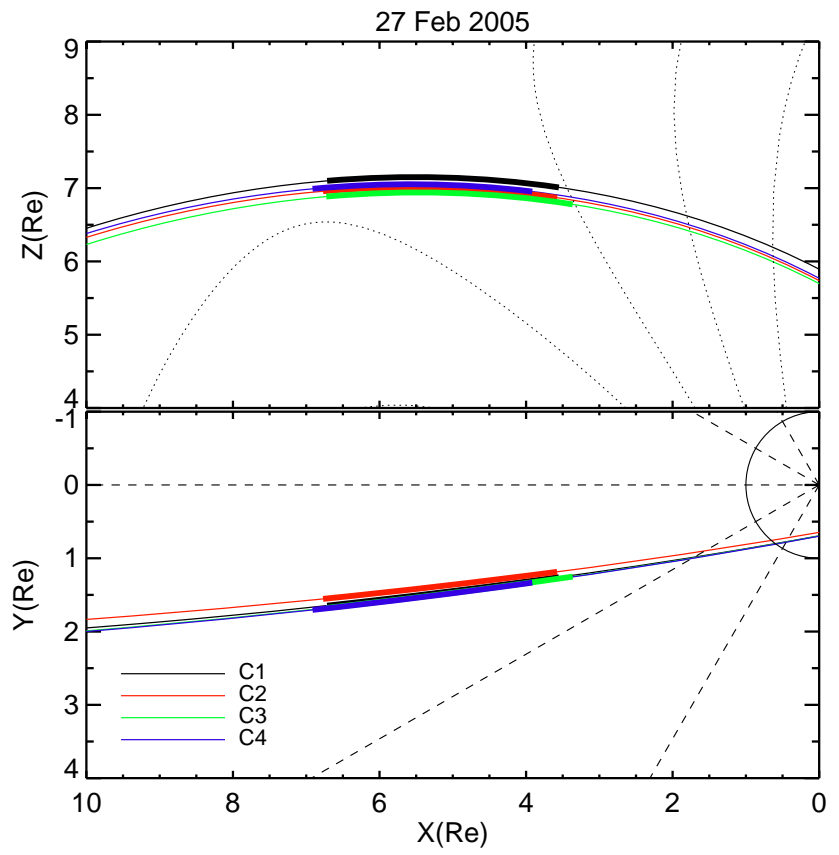

Fig. 1. The orbital projections of Cluster in GSM are displayed. The bold region identifies when the spacecraft were within the exterior cusp. The dotted lines in the top panel show the TS04 magnetic field model (Tsyganenko and Sitnov, 2005) for reference.

cusp (Lin et al., 2007) showing these ions have access to the cusp. Further evidence was provided by Trattner et al. (2001) who analyzed the ion abundance ratios and concluded these were also consistent with what would be expected with the bow shock. If the quasi-parallel bow shock is the primary source for energetic ions in the cusp and local energization is not occurring, an additional source would have to provide the observed energetic electron or ionospheric ion populations. It has been suggested that these populations could come from magnetospheric leakage at the magnetopause (Sibeck et al., 1987) or through particles drifting to the dayside high latitude region from the nightside (Blake et al., 1999).

The role of local acceleration of ions in the exterior cusp has been a topic of recent scientific discussion, however the role this region may play in energizing electrons up to hundreds of $\mathrm{keV}$ has received less attention. In this study we use a case study to analyze the properties of the energetic electron population in the cusp and test three potential sources for the energetic electrons: (1) solar energetic electrons; (2) outer equatorial ring current and radiation belt electrons; and (3) electrons energized through local acceleration.

\section{Observations: 27 February 2005}

On 27 February 2005, all four Cluster spacecraft crossed through the northern exterior cusp on an outbound pass. The position of the spacecraft as they passed through the CDC are shown in Fig. 1. The CDC is defined with the follow criteria: (1) magnetic variability identified by $\Delta B / B>20 \%$; (2) a depression of magnetic field strength of greater than $20 \%$ when compared with adjacent magnetospheric regions; and (3) stagnated or disturbed bulk flow.

Although all four spacecraft passed through the CDC during this event, we focus on the observations by $\mathrm{C} 3$ because it is the only one with a full set of observations from each instrument during the entire time period of interest. The plasma and energetic ion observations from $\mathrm{C} 3$ are shown in Fig. 2. Plasma moments were acquired through the Hot Ion Analyzed (HIA) as part of the CIS instrument (Rème et al., 1997, 2001), the energetic ions were measured with RAPID (Wilken et al., 1997, 2001), and the magnetic field measurements were measured with FGM (Balogh et al., 1997, 2001). At 17:49 UT C3 transitions from the northern lobe to the exterior cusp. At this time, the magnetic field strength decreased and became turbulent, and the plasma density increased by an order of magnitude from 1 to $10 \mathrm{~cm}^{-2}$. Inside the CDC, the $T_{\perp}$ and $T_{\|}$components were similar. The bulk flow components were low and representative of the stagnant exterior cusp (SEC) described by Lavraud et al. (2002, 2004). At 19:47 UT C3 crossed the magnetopause into the magnetosheath. The boundary crossing is identified by an increase in bulk flow particularly in the $V_{\mathrm{x}}$ and $V_{\mathrm{z}}$ components. Both the perpendicular and parallel components of the temperature decreased and the $T_{\perp}$ component became dominant. The magnetic field strength in the magnetosheath increased and became less variable compared to that observed within the cusp.

The electron population observed by $\mathrm{C} 3$ is shown in Fig. 3 . Both Figs. 3 and 2 display the same time period. The energetic part of the population, with energies from $37-400 \mathrm{keV}$ was measured with RAPID. The lower energy portion of the population from $22 \mathrm{eV}-26.4 \mathrm{keV}$ was measured with PEACE (Johnstone et al., 1997). The electron flux shows large enhancements while inside the exterior cusp which cut off quickly once the spacecraft crossed the magnetopause. There is no obvious energy dispersion in the flux. The pitch angle distribution for electrons with energies between $37 \mathrm{keV}$ and $51 \mathrm{keV}$ was measured by RAPID and is shown in panel (b) of Fig. 3. Since the magnetic field strength in the magnetosheath is larger than that in the CDC, and we know the magnetic field strength will increase going down the throat of the cusp, there is a magnetic trap within the exterior cusp. Assuming a magnetic field strength of $43 \mathrm{nT}$ in the magnetosheath and using Eq. (1),

$\alpha_{\mathrm{c}}=\sin ^{-1}\left(\sqrt{B / B_{\text {mirror }}}\right)$

the trapped portion of the distribution can be identified. In Eq. (1) $B_{\text {mirror }}$ is the magnetic field strength at the mirror point, $\alpha_{\mathrm{c}}$ is the local cut off pitch angle for a trapped particle and $B$ is the local magnetic field strength. The black line over plotted in panel (b) of Fig. 3 shows $\alpha_{\mathrm{c}}$ and outlines 


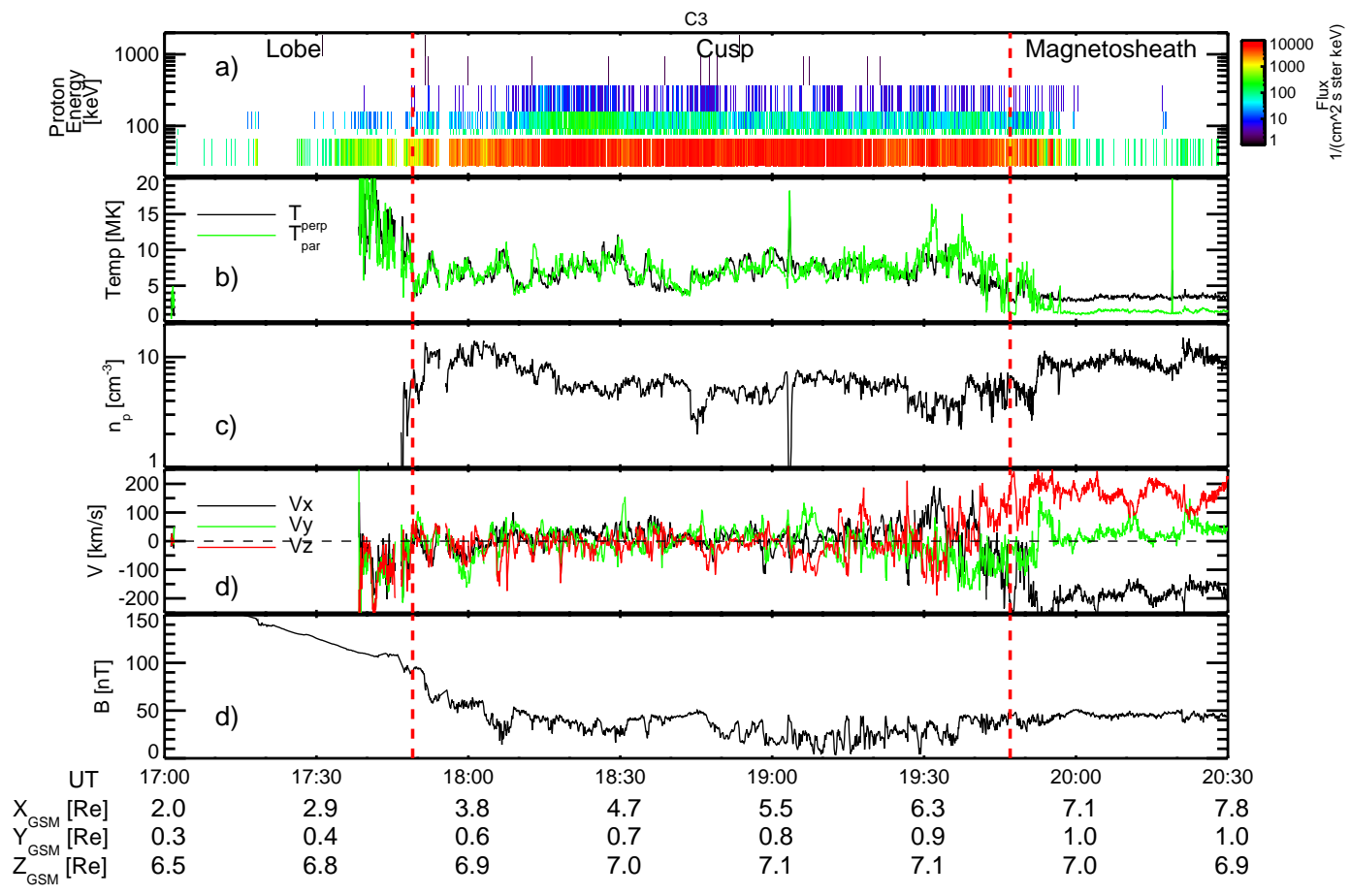

Fig. 2. Observations by C3 while passing through the exterior cusp. Panel (a) is energetic ion measurements from RAPID. The plasma moments in panels (b), (c), and (d) were measured by HIA. Panel (d) is the magnetic field strength measured by FGM. The vertical dashed lines identify where we have defined the spacecraft transition between the different magnetospheric regions.

the trapped portion of the population. Particles that are between the black line and $90^{\circ}$ are trapped. The majority of the energetic electron flux falls within these trapping limits. This format of overlaying a trapping limit onto a pitch angle time series was first shown by Nykyri et al. (2009). Nykyri et al. (2009) showed that assuming conservation of the first adiabatic invariant for the cusp observations by Cluster on 14 February 2003, the energetic electrons observed may not directly originate from the magnetosheath or the magnetosphere. The current work presents a different case study and investigates the expected characteristics of each of the proposed sources for energetic electrons in the exterior cusp.

The phase space density was calculated using the flux measurements from the RAPID and PEACE detector to determine the possible connection of the cusp electron population with the adjacent regions (Fig. 4). The phase space density as a function of the first adiabatic invariant $(\mu)$ shows the phase space density of the electrons to be much higher in the exterior cusp than in the lobe or magnetosheath.

The solar wind IMF varied in magnitude but was primarily northward with a positive $B_{\mathrm{y}}$ and a negative $B_{\mathrm{x}}$ component. The upstream conditions measured by WIND and time propagated to the magnetopause are shown in Fig. 5. The $V_{\mathrm{x}}$ component of the solar wind was relatively steady, growing gradually from $533 \mathrm{~km} / \mathrm{s}$ to $560 \mathrm{~km} / \mathrm{s}$. The dynamic pressure gradually increases as well from 2.0 to $3.2 \mathrm{nPa}$. Some of the variation in energetic electron flux observed by $\mathrm{C} 3$ (panel a, Fig. 3) may have been the result of the cusp moving over the spacecraft due to variable IMF conditions. The first observations of enhanced electron flux within the cusp near 17:51 UT and again at 17:56 UT appear to be in a region where the electrons are not geomagnetically trapped within the cusp. The magnetic field strength (panel d, Fig. 3) changes significantly as the electron flux becomes enhanced and dissipates as well, indicating that the variation in flux is a result of cusp motion or compression. As the cusp moves or is compressed the spacecraft is observing different regions with different geomagnetic field strengths.

\section{Potential sources}

With the observations of this event, we can test the three possible sources for the energetic electrons observed in the magnetospheric cusp. Electrons originating from each source may have access to the cusp, however the characteristics of the electrons in the cusp and adjacent regions are different from different sources. By analyzing these signatures, we are able to identify which source is the main contributor to the energetic electron population in the cusp during this event. 


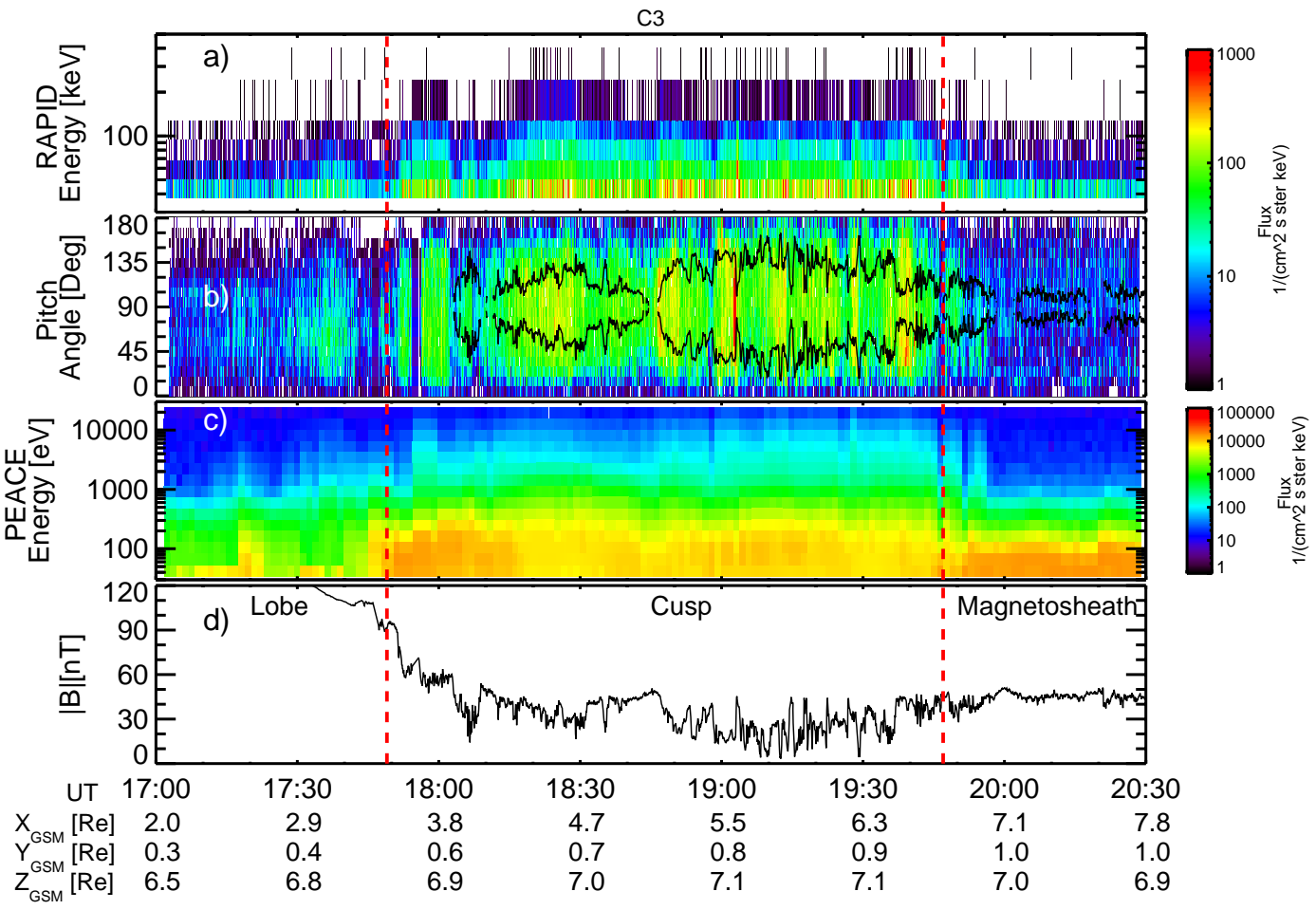

Fig. 3. Electron and magnetic field measurements by C3. Panel (a) is omnidirectional flux of energetic electrons from $37 \mathrm{keV}-400 \mathrm{keV}$ measured by RAPID. Panel (b) gives flux of electrons from $37-51 \mathrm{keV}$ with pitch angle as measured with the L3DD mode on RAPID. The black line overplotted in panel (b) shows the cutoff pitch angle which would trap electrons within the cusp. Panel (c) in omnidirectional electron flux from $22 \mathrm{eV}-26.4 \mathrm{keV}$ as measured by PEACE. The magnetic field strength is given in panel d. The vertical dashed lines identify where we have defined the spacecraft transition between the different magnetospheric regions.

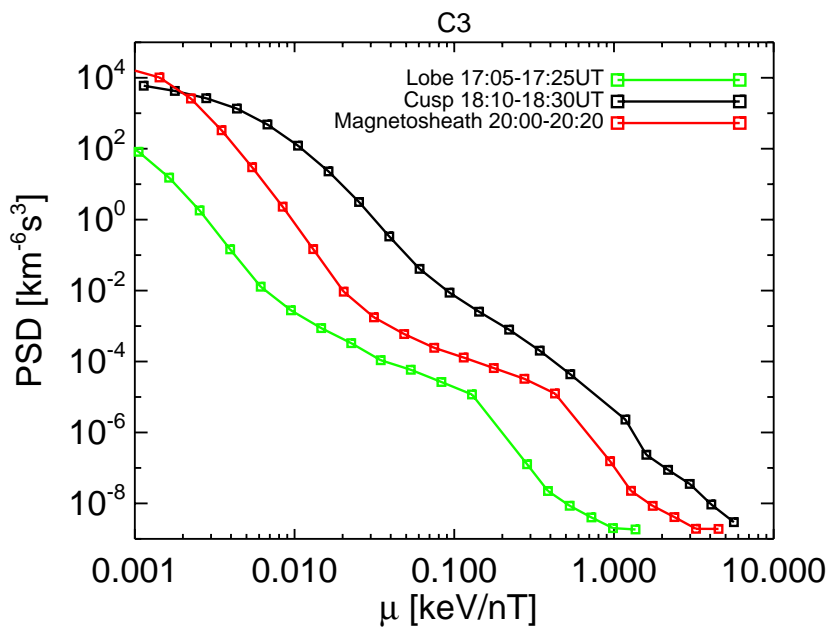

Fig. 4. Electron phase space density measured in the cusp, lobe, and magnetosheath as a function of the first adiabatic invariant. The colors green, black and red display the phase space density measured in the lobe, cusp and magnetosheath respectively.

\subsection{Solar source}

Energetic electrons propagating from the sun could have access to the cusp, however one would expect to observe them upstream before observing them in the cusp. Upstream observations by from the Electron, Proton, and Alpha Monitor (EPAM) (Gold, 1998) on ACE from 10:00 to 20:00 UT on 27 February 2005 reveal no energetic electron enhancements at four bands ranging from 38 to $315 \mathrm{keV}$ (Fig. 7). During this time period ACE was primarily upstream, with its location at $(\mathrm{X}, \mathrm{Y}, \mathrm{Z})=(218.8,11.1,-0.6) R_{\mathrm{E}}$ in GSE. Since there are no observed upstream flux enhancements during, and leading up to the event, it does not appear to be a reasonable source for this event.

The pitch angle distribution in the exterior cusp is also inconsistent with a solar source. Since the magnetic field in the magnetosheath is larger than that in the cusp, a pitch angle distribution would become more field aligned when entering a region of lower magnetic field to conserve the first adiabatic invariant. The energetic electrons in the exterior cusp are primarily at a pitch angle of $90^{\circ}$, therefore solar electrons can not be the primary source for the energetic population in the cusp. 


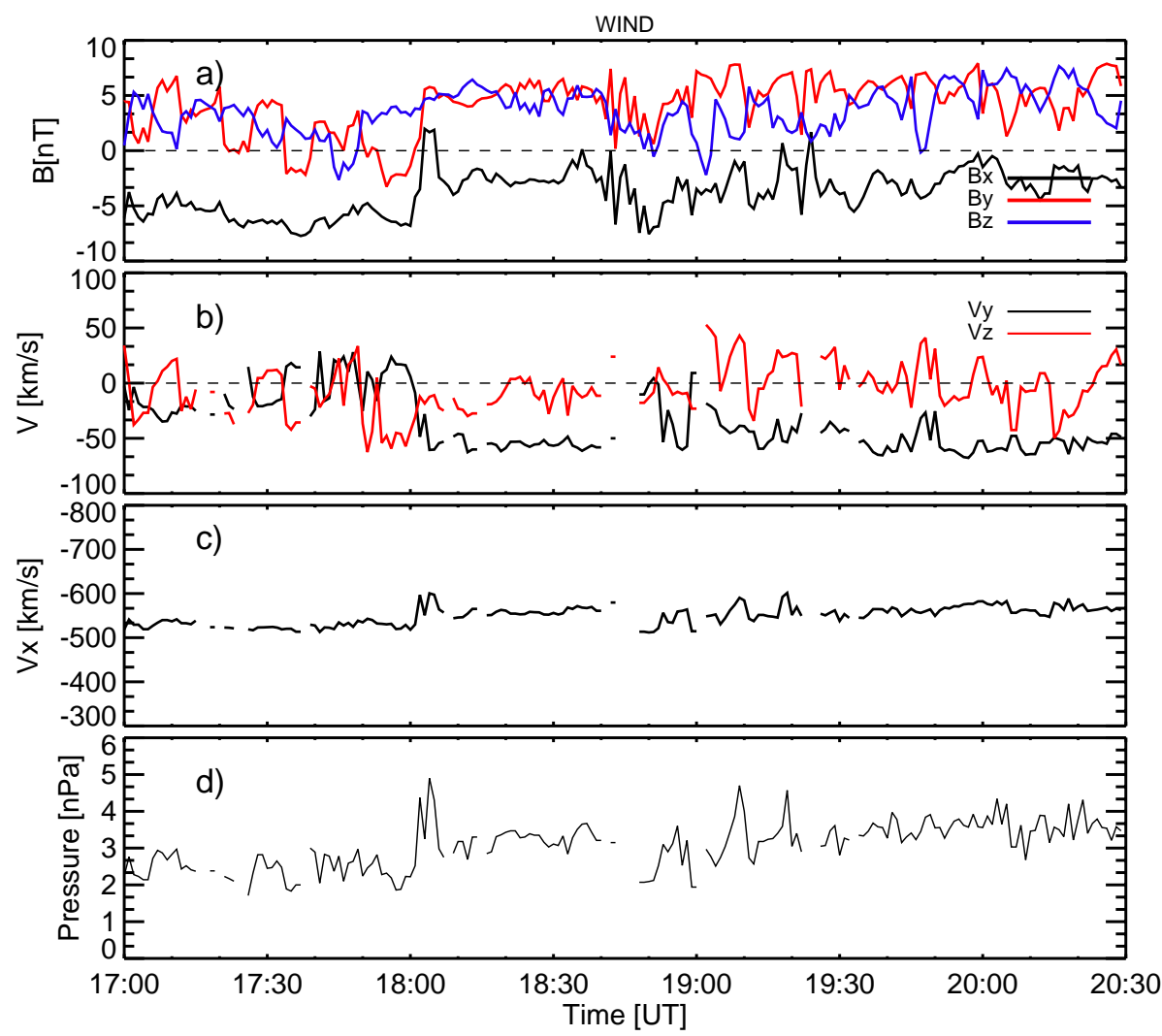

Fig. 5. Solar wind conditions measured by WIND and propagated to the magnetopause. Panel (a) is the magnetic field components in GSM. Panels (b) and (c) are the bulk plasma flow components. The dynamic pressure is given in panel (d).

Since the magnetic field strength is variable during this cusp crossing, the role of pitch angle scattering must also be considered. Whether the magnetic field seen by the electron is varying due to cusp motion, wave activity, or a magnetic null associated with reconnection traveling through the cusp, a turbulent magnetic field could cause the first adiabatic invariant to be violated and subsequently modify the particle's pitch angle. A $40 \mathrm{keV}$ electron similar to that shown in the pitch angle distribution (PAD) in panel (b) of Fig. 3 travels at roughly $18.5 R_{\mathrm{E}} / \mathrm{s}$. This means if pitch angle scattering is to scatter a field aligned electron traveling from another source through the cusp, it needs to be able to efficiently scatter the pitch angle in a fraction of a second. The role pitch angle scattering is playing can be determined to an extent by looking at the PAD as shown in Fig. 6. If strong scattering is playing a significant role and pitch angles are being scattered randomly, one would expect an isotropic PAD. Since the PAD in Fig. 6 shows a strong peak at $90^{\circ}$ with a loss cone near $0^{\circ}$ and $180^{\circ}$, it is unlikely the distribution has been heavily influenced by strong pitch angle scattering. From the observed PAD, a particle flowing through the cusp in a fraction of a second is unlikely to become scattered into the trapped portion of the PAD.
Lastly, the phase space density in the cusp is higher than that in the magnetosheath as shown in Fig. 4. If the electrons were entering the cusp via the magnetosheath, the phase space density in the magnetosheath would be the same or higher than the cusp. Invoking Liouville Theorem, this means the electron population in the cusp can not be coming from the magnetosheath at this time.

In agreement with this case study, a statistical study by Formisano (1979) of $40 \mathrm{keV}-2 \mathrm{MeV}$ electrons in the magnetosheath and along the high latitude magnetopause has shown the electrons in this region primarily come from the cusp. This was demonstrated through three main observations: (1) electron flux dropped off with distance from the cusp; (2) with increasing distance from the cusp, the electron energy spectrum hardened; and (3) the electron anisotropy and the magnetic fields indicate that the electrons are propagating away from the cusp along magnetic field lines. With our case study and the previous statistical work, we conclude that solar energetic electrons are not a primary source for energetic electrons in the cusp. 


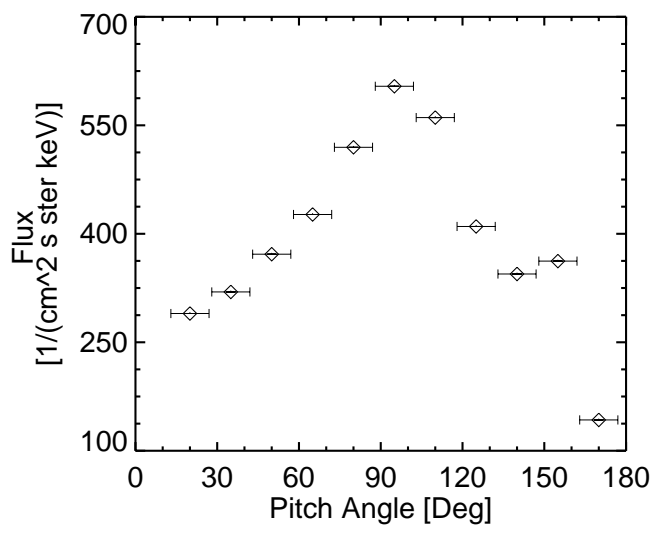

Fig. 6. Pitch angle distribution for electrons from $37-51 \mathrm{keV}$ measured by RAPID on $\mathrm{C} 3$. The measurements were taken during the time period 18:40-18:45 UT. The pitch angle bin size is $15^{\circ}$ and is shown by the error bars.

\subsection{Equatorial source}

Interaction of the solar wind with the geomagnetic field causes a compression at the dayside magnetopause. As a result of the compression, the equatorial minimum in the geomagnetic field bifurcates and moves to high latitudes for large L-shells (Mead, 1964). Particles drifting through these L-shells will subsequently travel to the high latitudes on the dayside rather than simply drifting along the equator (Antonova and Shabansky, 1968; Shabansky, 1968). Delcourt and Sauvaud $(1998,1999)$ have used single particle traces to show that rather than being stably trapped, energetic ions from the outer radiation belt and ring current that move to high latitudes on the dayside are dynamically trapped for short time periods and are then scattered back to the night side. Simulations of energetic electrons in the high latitude dayside region and in the absence of an electric field have shown similar results with large stable trapping regions covering a broad area (Zong et al., 2005).

To test the flexibility of a particle trajectory that could lead energetic electrons to the high-latitude dayside, we modeled the drift path of energetic electrons with a fully relativistic three-dimensional particle trace. The full Lorentz force is solved with a 4th order Runge-Kutta method to propagate the particles. The TS04 magnetic field model (Tsyganenko and Sitnov, 2005) as well as the equatorial Volland-Stern electric field model (Stern, 1975; Volland, 1978) are used. The electric field vector at positions off the equator was found by treating the magnetic field lines as equipotentials and propagating the Volland-Stern vector along the magnetic field line. Given a particle's initial mass, charge, energy, location, pitch angle, and gyrophase, the tracer can model a particle's progression in the magnetosphere.

We confirmed that energetic electrons starting within a narrow equatorial region on the night side will drift to a bi-

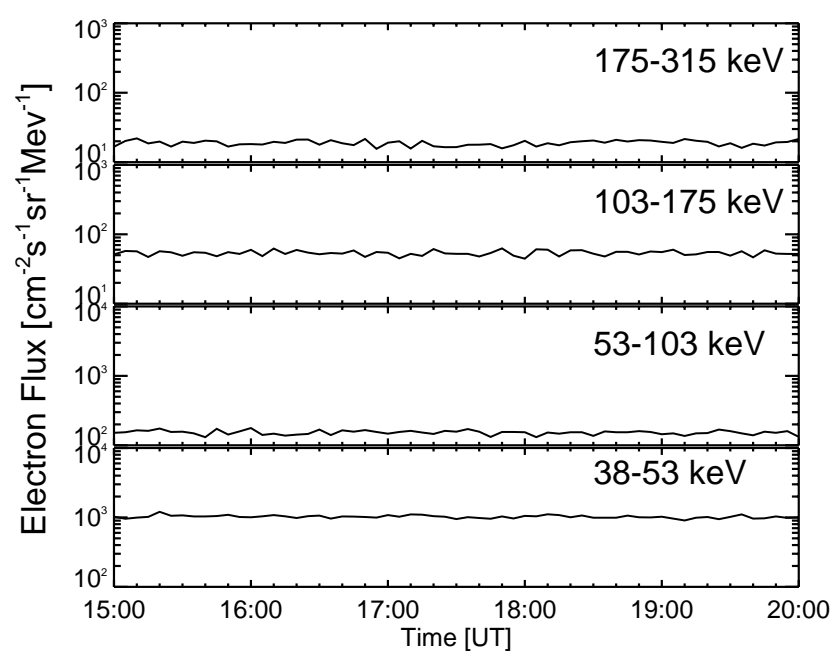

Fig. 7. Upstream energetic electron observations from EPAM on ACE from 27 February 2005. Each energy channel is displayed with a 5 min time cadence.

furcation point on the dayside and populate the high-latitude dayside either in the Northern or Southern Hemisphere. The values input to the electric and magnetic field models were $\mathrm{kp}=3, n_{\mathrm{sw}}=4.85 \mathrm{~cm}^{-2}$, Dst $=-3 \mathrm{nT}, v_{\mathrm{sw}}=538 \mathrm{~km} / \mathrm{s}$, $B_{\mathrm{y}}=2.8 \mathrm{nT}, B_{\mathrm{z}}=3.2 \mathrm{nT}$. Using these conditions for the 27 February 2005 event, a $200 \mathrm{keV}$ electron with an initial pitch angle of $90^{\circ}$ and starting at GSM $(\mathrm{X}, \mathrm{Y}, \mathrm{Z})=(-10.5,0.0$, 0.0) $R_{\mathrm{E}}$ will drift to the high latitude dayside. The electron will continue around the contour of constant $B$ at highlatitude and then drift back to the equator. The nightside equatorial region tracing to the high-latitude dayside is relatively narrow as the same $200 \mathrm{keV}$ electron starting at (X, Y, $\mathrm{Z})=(-9.5,0.0,0.0) R_{\mathrm{E}}$ will not bifurcate and will simply drift eastward along the equator and back to the nightside. Additionally, a $200 \mathrm{keV}$ electron starting at $(\mathrm{X}, \mathrm{Y}, \mathrm{Z})=(-11.5,0.0$, $0.0) R_{\mathrm{E}}$ under the same solar wind conditions will drift into the magnetopause compressed on the dayside and will be lost from the system. These drift paths are shown in Fig. 8.

Sheldon et al. (1998) modeled energetic electrons through the high latitude dayside minimum and found it to be a steady trapping region without the presence of an electric field. Our results are more consistent with the prediction of Delcourt and Sauvaud (1999) who incorporated an electric field and showed energetic ions that drift to the high latitude dayside are dynamically trapped and will eventually return to the equator.

With knowledge of how the electrons drift to the high latitude dayside, we can test if this is consistent with the observations. If the equatorial electrons populate a wide portion of the high-latitude region, as shown in the particle trace (Fig. 8), and are the primarily source for the electrons observed in the cusp, the phase space density of the cusp electrons should be similar to or the same as in the adjacent 
magnetospheric regions. Fig. 4 shows the phase space density in the cusp is much higher than the lobe region therefore they can not be from the same source.

Another reason a nightside equatorial source is inconsistent with the observations is that there is no apparent energy dispersion in the electron flux. A packet of electrons injected from the night side and drifting to the dayside high latitude would drift at different rates and produce an energy dispersion when observed in the cusp. This effect has been observed and modeled with electrons injected on the nightside and drifting to other regions of the magnetosphere (Lutsenko et al., 2000, 2005). The observed energetic electron flux shows no obvious energy dispersion (panel a, Fig. 3), therefore electrons from the radiation belt can not be the primary source for the energetic electrons in the exterior cusp during this event.

Asikainen and Mursula $(2005,2006)$ proposed a variation to the nightside source. Rather than drifting to the cusp (Blake et al., 1999; Delcourt and Sauvaud, 1999), Asikainen and Mursula $(2005,2006)$ suggested electrons could be released from the high latitude plasma sheet (HLPS) equatorward of the cusp to the exterior cusp via reconnection. Flux transfer event (FTE) signatures have also been observed with connections to bursts of energetic electrons (Zong et al., 2003; Asikainen and Mursula, 2006). These bursts of energetic electrons with FTE give evidence that when the geometry is appropriate, electrons can be released from the HLPS into the cusp. These events can be identified from the bursty nature of the flux and the electron pitch angle distributions. A $200 \mathrm{keV}$ electron travels at roughly $40 R_{\mathrm{E}} / \mathrm{s}$, so particles entering the cusp via reconnection will bounce out of the cusp quite quickly and a show little flux between bursts. The pitch angle distribution of electrons released from the HLPS would be close to field aligned in the exterior cusp since the magnetic field strength in the HLPS is greater than that of the cusp. The observations of energetic electrons during the 27 February 2005 event show a trapped pitch angle distribution centered around $90^{\circ}$ for almost two hours and is therefore inconsistent with electrons released to the cusp from the HLPS. Energetic electrons coming from the HLPS are likely to contribute to the population under some conditions, however, this event shows observations where the bulk of energetic population is not consistent with a HLPS source.

During the current study, the IMF $B_{\mathrm{Z}}$ component remained positive for the entire event with the exception of a several minute period near 19:00 UT, while the IMF $B_{\mathrm{y}}$ remains positive after 18:00 UT (Fig. 5). In the frame of antiparallel reconnection, the reconnection site would be poleward of the cusp, and the cusp location would move towards the duskside in the Northern Hemisphere due to the IMF conditions (Crooker, 1979). The magnitudes of the components are variable, however while the IMF $B_{\mathrm{Z}}$ component remains positive, the reconnection site is anticipated to remain polward of the cusp. Reconnection in this location would cause two reconnected flux tubes, one moving equatorward that could

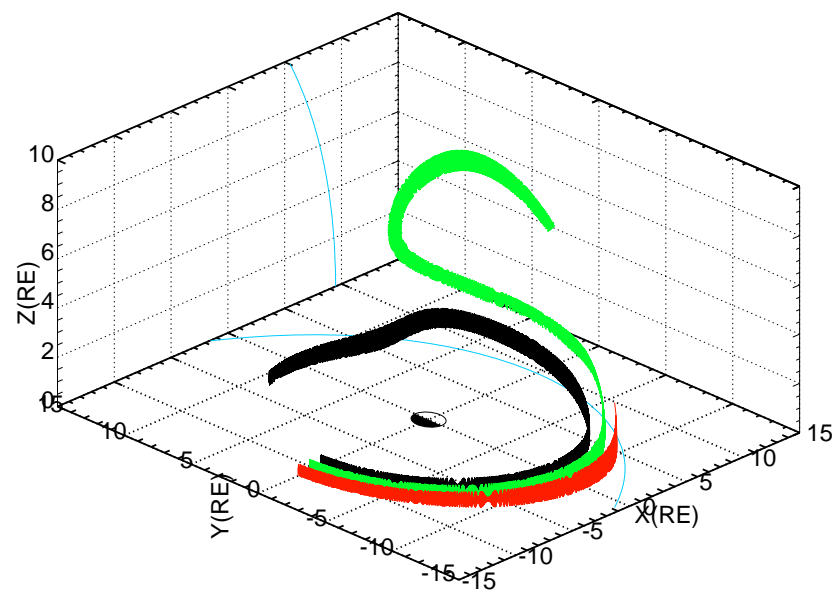

Fig. 8. Electron $(E=200 \mathrm{keV})$ trajectories traced in a TS04 model magnetic field (Tsyganenko and Sitnov, 2005) and a Volland-Stern electric model (Stern, 1975; Volland, 1978). Each electron is released with a gyrophase of 0 and a pitch angle of $90^{\circ}$. The black trace is an electron released at GSM $(\mathrm{X}, \mathrm{Y}, \mathrm{Z})=(-9.5,0,0) R_{\mathrm{E}}$ which drifts around the equator and returns to the night side. The green trace is an electron released at $(\mathrm{X}, \mathrm{Y}, \mathrm{Z})=(-10.5,0,0) R_{\mathrm{E}}$ that moves to high latitude on the dayside and drifts around the cusp. Lastly, the red trace is an electron released at $(\mathrm{X}, \mathrm{Y}, \mathrm{Z})=(-11.5$, $0,0) R_{\mathrm{E}}$ that collides with the magnetopause on the dayside and is lost from the system. The light blue lines show a modeled magnetopause (Shue et al., 1998) drawn for reference.

pass through the cusp and one moving tailward. Cluster passed through the lobe region prior to the cusp and observed very low energetic electron flux (panel a, Fig. 3). Since very few energetic electrons were observed in the lobe, an FTE traveling from a reconnection site poleward of the cusp to the cusp would not deliver energetic electrons as is sometimes seen during time periods with reconnection equatorward of the cusp (Zong et al., 2003; Asikainen and Mursula, 2006).

\subsection{Local source}

A local acceleration source seems to be consistent with the observations, and therefore is likely the primary source for energetic electrons in the exterior cusp for this event. If local acceleration was the dominant source, one would expect a higher phase space density in the cusp than in adjacent regions. This was observed and is shown in Fig. 4. The phase space density for electrons with $E>100 \mathrm{eV}$ and up to the energy limit of the RAPID detector $(400 \mathrm{keV})$, is significantly larger in the cusp than in the lobe and magnetosheath adjacent to the cusp. A local or near by source would also show no time energy dispersion which is observed and can be seen in Fig. 3, panel (a). The pitch angle distribution also implies a trapped energetic distribution within the CDC. This is inconsistent with electrons entering from an adjacent region with a stronger magnetic field strength. Panel (b) of Fig. 3 shows 
the majority of the electron flux is within the cut off trapping pitch angle. All of these signatures indicate significant local acceleration of the electrons up to several hundreds of $\mathrm{keV}$.

There have been several mechanisms suggested to be capable of accelerating the particles within this region. Chen (2008) showed that the large fluctuations in magnetic and electric fields observed in the cusp are often sufficient to energize particles up to several hundred $\mathrm{keV}$ in seconds through cyclotron resonance. Test particle simulations by Otto et al. (2007) have shown the potential formed due to reconnection under northward IMF can also accelerate particles efficiently. The magnetic geometry provides a trap for particles which can drift around the diamagnetic cavity and through the potential, gaining energy. Both mechanisms would primarily energize particles with perpendicular energy which is consistent with the electron population observed in this case study.

\section{Potential magnetospheric impact}

A layer of energetic electrons primarily above $40 \mathrm{keV}$ and up to several $\mathrm{MeV}$ has been shown to be a consistent feature along the high latitude magnetopause from the region just poleward of the cusp down the magnetotail (Meng and Anderson, 1970, 1975). The electrons in this layer are flowing tailward and show a peak in flux near the cusps (Domingo et al., 1977). These are indicators this population originates within or streams through the cusp. Baker and Stone (1977) emphasized the importance of this population by showing this magnetopause electron layer carried significant current and energy downtail. If electrons are being accelerated in the cusp to several hundred $\mathrm{keV}$, they could be a major source of the magnetopause electron layer. Although the 27 February 2005 event presented in this paper shows a population primarily trapped within the CDC, a shift in magnetospheric configuration or a change in magnetosheath magnetic field strength to levels closer to that in the cusp would cause electrons to flow out of the cusp into the magnetosheath and along the magnetopause.

The spectral characteristics of the cusp population also appear to be similar to that downstream along the magnetopause. During the time period between 18:20 and 18:30 UT on 27 February 2006, the flux distribution between 40 and $400 \mathrm{keV}$ is represented by a power law with a slope of $\alpha=-1.80$ where Flux $\sim A \cdot E^{\alpha}, E$ is energy and $A$ is a constant. Meng and Anderson (1975) measured a power law with a slope of $\alpha=-1.94 \pm 0.19$ for electrons downstream along the magnetopause. It's likely that local acceleration of electrons in the cusp can contribute to the electron population flowing downtail along the magnetopause.

Parts of the energized population along the magnetopause will subsequently gain access into the plasma sheet and magnetotail along the flanks due to gradient drift entry (GDE) (Olson and Pfitzer, 1984). The exact role GDE may play in allowing solar wind particles to cross the magnetopause how- ever is unclear. Olson and Pfitzer (1985) proposed that most of the shocked solar wind population striking the magnetopause could cross while Treumann and Baumjohann (1988) suggested that just $5 \%$ of the magnetosheath plasma could penetrate. What is clear however, is that the energetic electrons observed in the cusp and then encountering the magnetopause will have a greater ability to cross the magnetopause via GDE than the magnetosheath plasma which typically contains fewer energetic particles. GDE is more efficient for particles of with larger gyroradii, so the more energetic particles will have more access to the plasma sheet and magnetotail.

This access into the magnetosphere via GDE also changes with IMF direction. During periods of northward IMF, when the dawn-dusk electric field is small, the access is enhanced for particles of all energies, while under southward IMF, a velocity filter exists that prevents less energetic particles from crossing the magnetopause (Zhou et al., 2005). Zhou et al. (2005) has suggested this differences between the GDE efficiency during northward and southward IMF could be related to the formation of the plasma sheet. The cold and dense plasma sheet generally observed during northward IMF versus the hot and tenuous plasma sheet observed during southward IMF are consistent with the populations one would expect to be crossing the magnetopause by GDE during each IMF orientation. If acceleration in the exterior cusp is efficient enough to be contributing to the energetic particle population along the magnetopause, it could play a role in enhancing the GDE and populating the plasma sheet.

\section{Conclusions}

Through analyzing properties of energetic $(E>40 \mathrm{keV})$ electrons in the exterior cusp measured by Cluster on 27 February 2005, we have determined local energization is the primary source of this population. We compared spacecraft observations by $\mathrm{C} 3$ with expected measurements for (1) a solar energetic electron source, (2) an equatorial outer ring current or radiation belt source and (3) local energization. It is expected that all three sources have access to the cusps, however each one would show different characteristics in the cusp and adjacent magnetospheric regions. Local energization is the only source that is fully consistent with the measurements, thus we select it as the primary source of the energetic electron population in the exterior cusp during this event. Although other sources may contribute to the energetic population within the cusp under certain conditions, this event serves as evidence that the cusp is capable of accelerating large amounts of particles to energies of several hundred $\mathrm{keV}$.

Acknowledgements. We would like to acknowledge the Cluster Active Archive and their open data policy that was used in this study. This work was supported by the NASA grant NNX08AU70G. 
Topical Editor R. Nakamura thanks two anonymous referees for their help in evaluating this paper.

\section{References}

Antonova, A. E. and Shabansky, V. P.: Structure of the geomagnetic field at great distance from the Earth, Geomagn. Aeron., 8, 801811,1968

Asikainen, T. and Mursula, K.: Energetic particle fluxes in the exterior cusp and the high-latitude dayside magnetosphere: statistical results from the Cluster/RAPID instrument, Ann. Geophys., 23, 2217-2230, 2005, http://www.ann-geophys.net/23/2217/2005/.

Asikainen, T. and Mursula, K.: Reconnection and energetic particles at the edge of the exterior cusp, Ann. Geophys., 24, 19491956, 2006, http://www.ann-geophys.net/24/1949/2006/.

Baker, D. N. and Stone, E. C.: The Magnetopause Electron Layer along the Distant Magnetotail, Geophys. Res. Lett., 4, 395-398, 1977.

Blake, J. B.: Comment on 'Cusp: A new Acceleration Region of the Magnetosphere' by Jiasheng Chen et al., Czech. J. Phys. 49(4a), 675-677, 1999

Balogh, A., Dunlop, M. W., Cowley, S. W. H., et al.: The Cluster Magnetic Field Investigation, Space Sci. Rev., 79, 65-91, 1997.

Balogh, A., Carr, C. M., Acuña, M. H., Dunlop, M. W., Beek, T. J., Brown, P., Fornacon, K.-H., Georgescu, E., Glassmeier, K.H., Harris, J., Musmann, G., Oddy, T., and Schwingenschuh, K.: The Cluster Magnetic Field Investigation: overview of in-flight performance and initial results, Ann. Geophys., 19, 1207-1217, 2001, http://www.ann-geophys.net/19/1207/2001/.

Chang, S.-W., Scudder, J. D., Fuselier, S. A., Fennell, J. F., Trattner, K. J., Pickett, J. S., Spence, H. E., Menietti, J. D., Peterson, W. K., Lepping, R. P., and Friedel, R.: Cusp energetic ions: A bow shock source, Geophys. Res. Lett., 25, 3729-3732, 1998.

Chen, J., Fritz, T. A., Sheldon, R. B., Spence, H. E., and Spjeldvik, W. N.: A new, temporarily confined population in the polar cap during the August 27, 1996 geomagnetic field distortion period, Geophys. Res. Lett., 24, 1447-1450, 1997.

Chen, J. and Fritz, T. A.: Correlation of cusp MeV helium with turbulent ULF power spectra and its implications, Geophys. Res. Lett., 25, 4113-4116, 1998.

Chen, J., Fritz, T. A., Sheldon, R. B., Spence, H. E., Spjeldvik, W. N., Fennell, J. F., Livi, S., Russell, C. T., Pickett, J. S., and Gurnett, D. A.: Cusp energetic particle events: Implications for a major acceleration region of the magnetosphere, J. Geophys. Res., 103(A1), 69-78, 1998.

Chen, J. and Fritz, T. A.: Energetic oxygen ions of ionospheric origin observed in the cusp, Geophys. Res. Lett., 28, 1459-1462, 2001.

Chen, J.: Evidence for particle acceleration in the magnetospheric cusp, Ann. Geophys., 26, 1993-1997, 2008, http://www.ann-geophys.net/26/1993/2008/.

Crooker, N. U.: Dayside merging and cusp geometry, J. Geophys. Res., 84, 951-959, 1979.

Delcourt, D. C. and Sauvaud, J. A.: Recirculation of plasma sheet particles into the high-latitude boundary layer, J. Geophys. Res. 103, 26521-26532, 1998.

Delcourt, D. C. and Sauvaud, J.-A.: Populating of the cusp and boundary layers by energetic (hundreds of $\mathrm{keV}$ ) equatorial particles, J. Geophys. Res., 104(A10), 22635-22648, 1999.
Domingo, V., Page, D. E., and Wenzel, K. -P.: Energetic and Relativistic Electrons Near the Polar Magnetopause, J. Geophys. Res., 82(16), 2327-2326, 1977.

Farrell, W. M. and Van Allen, J. A.: Observations of the earth's polar cleft at large radial distances with the Hawkeye 1 magnetometer, J. Geophys. Res., 95, 20945-20958, 1990.

Fairfield, D. H. and Ness, N. F.: IMP 5 magnetic-field measurements in the high-latitude outer magnetosphere near the noon meridian, J. Geophys. Res., 77, 611-623, 1972.

Formisano, V.: Properties of Energetic Electrons of Magnetospheric Origin in the Magnetosheath and in the Solar Wind, Planet. Space Sci., 27, 867-879, 1979.

Frank, L. A.: Plasma in the Earth's polar magnetosphere, J. Geophys. Res., 76(22), 5202-5219, 1971.

Fritz, T. A., Chen, J., and Siscoe, G. L.: Energetic ions, large diamagnetic cavities, and Chapman-Ferraro cusp, J. Geophys. Res., 108, A1, doi:10.1029/2002JA009476, 2003.

Gold, R. E., Krimigis, S. M., Hawkins, S. E., Haggerty, D. K., Lohr, D. A., Fiore, E., Armstrong, T. P., Holland, G., and Lanzerotti, L. J.: Electron, Proton, and Alpa Monitor on the Advances Composition Explorer Spacecraft, Space Sci. Rev., 86, 541-562, 1998.

Heikkila, W. J. and Winningham, J. D.: Penetration of Magnetosheath Plasma to Low Altitudes though the Dayside Magnetospheric Cusp, J. Geophys. Res., 76, 883-891, 1971.

Johnstone, A. D., Alsop, C., Burge, S., et al.: PEACE: A plasma electron and current experiment, Space Sci. Rev., 79, 351-398, 1997.

Khotyaintsev, Y., Vaivads, A., Ogawa, Y., Popielawska, B., André, M., Buchert, S., Décréau, P., Lavraud, B., and Rème, H.: Cluster observations of high-frequency waves in the exterior cusp, Ann. Geophys., 22, 2403-2411, 2004, http://www.ann-geophys.net/22/2403/2004/.

Lavraud, B., Dunlop, M. W., Phan, T. D., Rème, H., Bosqued, J.M., Dandouras, I., Sauvaud, J.-A., Lundin, R., Taylor, M. G. G. T., Cargill, P. J., Mazelle, C., Escoubet, C. P., Carlson, C. W., McFadden, J. P., Parks, G. K., Moebius, E., Kistler, L. M., Bavassano-Cattaneo, M.-B., Korth, A., Klecker, B., and Balogh, A.: Cluster observations of the exterior cusp and its surrounding boundaries under northward IMF, Geophys. Res. Lett., 29(20), 1995, doi:10.1029/2002GL015464, 2002.

Lavraud, B., Phan, T. D., Dunlop, M. W., Taylor, M. G. G. G. T., Cargill, P. J., Bosqued, J.-M., Dandouras, I., Rme, H., Sauvaud, J.-A., Escoubet, C. P., Balogh, A., and Fazakerley, A.: The exterior cusp and its boundary with the magnetosheath: Cluster multi-event analysis, Ann. Geophys., 22, 3039-3054, 2004, http://www.ann-geophys.net/22/3039/2004/.

Le, G., Blanco-Cano, X., Russell, C. T., et al.: Electromagnetic ion cyclotron waves in the high-altitude cusp: Polar observations, J. Geophys. Res., 106, 19067-19079, 2001.

Lin, Y., Wang, X. Y., and Chang, S.-W.: Connection between bow shock and cusp energetic ions, Geophys. Res. Lett., 34, L11107, doi:10.1029/2007GL030038, 2007.

Lutsenko, V. N., Grechko, T. V., and Kudela, K.: Interball-2 and -1 observations of energy dispersion events in Auroral zone for 30$500 \mathrm{keV}$ ions and electrons, Substorms 5, ESA SP-443, 519-522, 2000.

Lutsenko, V. N., Kirpichev, I. P., Grechko, T. V., and Delcourt, D.: Source positions of energetic particles responsible for the fine dispersion structures: numerical simulation results, Planet. Space 
Sci., 53, 275-281, 2005.

Mead, G. D.: Deformation of the geomagnetic field by the solar wind, J. Geophys. Res., 69(7), 1181-1195, 1964.

Meng, C. I. and Anderson, K. A.: A Layer of Energetic Electrons ( $>40 \mathrm{keV}$ ) near the Magnetopause, J. Geophys. Res., 75, 1827 1836.

Meng, C. I. and Anderson, K. A.: Characteristics of the Magnetopause Energetic Electrons Layer, J. Geophys. Res., 80, 42374243, 1975.

Nykyri, K., Cargill, P. J., Lucek, E., Horbury, T., Lavraud, B., Balogh, A., Dunlop, M. W., Bogdanova, Y., Fazakerley, A., Dandouras, I., and Rme, H.: Cluster observations of magnetic field fluctuations in the high-altitude cusp, Ann. Geophys., 22, 24132429, 2004, http://www.ann-geophys.net/22/2413/2004/.

Nykyri, K., Otto, A., Adamson, E. T., Kronberg, E., Daly, P. W.., and Lavraud, B.: On the Origin of High-Energy Electrons in Cusp Diamagnetic Cavities, AGU Fall Meeting, 2009.

Olson, W. P. and Pfitzer, K. A.: The entry of AMPTE lithium ions into a magnetically closed magnetosphere, J. Geophys. Res., 89, 7347-7350, 1984.

Olson, W. P. and Pfitzer, K. A.: Magnetospheric responses to the gradient drift entry of solar wind plasma, J. Geophys. Res., 90, 10823-10833, 1985.

Otto, A., Adamson, E., and Nykyri, K.: Acceleration in Cusp Diamagnetic Cavities, AGU Fall Meeting, 2007.

Paschmann, G., Haerendel, G., Sckopke, N., Rosenbauer, H., and Hedgecock, P. C.: Plasma and Magnetic Field Characteristics of the Distant Polar Cusp Near Local Noon: The Entry Layer, J. Geophys. Res., 81, 2883-2899, 1976.

Pickett, J. S., Franz, J. R., Scudder, J. D., et al.: Plasma waves observed in the cusp turbulent boundary layer: An analysis of high time resolution wave and particle measurements from the Polar spacecraft, J. Geophys. Res., 106, 19081-19100, 2001.

Rème, H., Bosqued, J. M., Sauvaud, J. A., et al.: The Cluster Ion Spectrometry (CIS) Experiment, Space Sci. Rev., 79, 303-350, 1997.

Rème, H., Aoustin, C., Bosqued, J. M., et al.: First multispacecraft ion measurements in and near the Earths magnetosphere with the identical Cluster ion spectrometry (CIS) experiment, Ann. Geophys., 19, 1303-1354, 2001, http://www.ann-geophys.net/19/1303/2001/.

Shabansky, V. P.: Magnetospheric Process and Related Geophysical Phenomena, Space Sci. Rev., 8, 366-454, 1968.

Sheldon, R. B., Spence, H. E., Sullivan, J. D., Fritz, T. A., and Chen, J.: The discovery of trapped energetic electrons in the outer cusp, Geophys. Res. Lett., 25, 1825-1828 1998.

Shue, J.-H., Song, P., Russell, C. T. , Steinberg, J. T., Chao, J. K., Zastenker, G., Vaisberg, O. L., Kokubun, S., Singer, H. J., Detman, T. R., and Kawano H.: Magnetopause location under extreme solar wind conditions, J. Geophys. Res., 103, 1769117710, 1998.

Sibeck, D. G., McEntire, R. W., Lui, A. T. Y., Lopez, R. E., Krimigis, A. M., Decker, R. B., Zanetti, L. J., and Potemra, T. A.: Energetic magnetospheric ions at the dayside magnetopause: Leakage or merging?, J. Geophys. Res., 92, 12097-12114, 1987.

Stern, D. P.: Charged particle motions in a magnetic field that reduce to motions in a potential, Am. J. Phys., 43, 689-694, doi:10.1119/1.9714, 1975.

Tsyganenko, N. A.: Magnetic field and electric currents in the vicin- ity of polar cusps as inferred from Polar and Cluster data, Ann. Geophys., 27, 1573-1582, 2009,

http://www.ann-geophys.net/27/1573/2009/.

Tsyganenko, N. A. and Sitnov, M. I.: Modeling the dynamics of the inner magnetosphere during strong geomagnetic storms, J. Geophys. Res., 110, 3208-3223, doi:10.1029/2004JA010798, 2005.

Trattner, K. J., Fuselier, S. A., Peterson, W. K., et al.: Origins of energetic ions in the cusp, J. Geophys. Res., 106, 5967-5976, 2001.

Treumann, R. A. and Baumjohann, W.: Particle trapping at a tangential discontinuity: Multiple incidence, Planet. Space Sci., 36, 1477-1484, 1988.

Vogiatzis, I. I., Sarris, T. E., Sarris, E. T., Santolík, O., Dandouras, I., Robert, P., Fritz, T. A., Zong, Q.-G., and Zhang, H.: Cluster observations of particle acceleration up to supra-thermal energies in the cusp region related to low-frequency wave activity possible implications for the substorm initiation process, Ann. Geophys., 26, 653-669, 2008,

http://www.ann-geophys.net/26/653/2008/.

Volland, H.: A model of the magnetospheric electric convection field, J. Geophys. Res., 83, 2695-2699, 1978.

Walsh, B. M., Fritz, T. A., Lender, N. M., Chen, J., and Whitaker, K. E.: Energetic particles observed by ISEE-1 and ISEE-2 in a cusp diamagnetic cavity on 29 September 1978, Ann. Geophys., 25, 2633-2640, 2007,

http://www.ann-geophys.net/25/2633/2007/.

Whitaker, K. E., Chen, J., and Fritz, T. A.: CEP population observed by ISEE 1, Geophys. Res. Lett., 33, L23105, doi:10.1029/2006GL027731, 2006.

Wilken, B., Axford, W. I., Daglis, I., et al.: RAPID: The Imaging Energetic Particle Spectrometer on Cluster, Space Sci. Rev., 79, 399-473, 1997.

Wilken, B., Daly, P. W., Mall, U., Aarsnes, K., Baker, D. N., Belian, R. D., Blake, J. B., Borg, H., Büchner, J., Carter, M., Fennell, J. F., Friedel, R., Fritz, T. A., Gliem, F., Grande, M., Kecskemety, K., Kettmann, G., Korth, A., Livi, S., McKenna-Lawlor, S., Mursula, K., Nikutowski, B., Perry, C. H., Pu, Z. Y., Roeder, J., Reeves, G. D., Sarris, E. T., Sandahl, I., Sraas, F., Woch, J., and Zong, Q.-G.: First results from the RAPID imaging energetic particle spectrometer on board Cluster, Ann. Geophys., 19, 1355-1366, 2001, http://www.ann-geophys.net/19/1355/2001/.

Zhang, H., Fritz, T. A., and Zong, Q.-G. : Stagnant exterior cusp region as viewed by energetic electrons and ions: A statistical study using Cluster Research with Adaptive Particle Imaging Detectors (RAPID) data, J. Geophys. Res., 110, A05211, doi:10.1029/2004JA010562, 2005.

Zhou, X.-Z., Pu, Z. Y., Zong, Q.-G., and Xie, L.: Energy filter effect for solar wind particle entry to the plasma sheet via flank regions during southward interplanetary magnetic field, J. Geophys. Res., 112, A06233, doi:10.1029/2006JA012180, 2007.

Zong, Q.-G., Fritz, T. A., Spence, H. E., Dunlop, M., Pu, Z. Y., Korth, A., Daly, P. W., Balogh, A., and Rème, H.: Bursty energetic electrons confined in Flux ropes in the cusp region, Planet. Space Sci., 51, 821-830, 2003.

Zong, Q.-G., Fritz, T. A., Korth, A., et al.: Energetic electrons as a field line topology tracer in the high latitude boundary/cusp region: Cluster/RAPID observations, Surv. Geophys., 26, 2150 240, 2005. 